\title{
Estudio comparativo sobre el desarrollo de proveedores en dos ramas industriales: automotriz y textil y de la confección
}

Pilar Ester

Arroyo López.

Profesora titular, Instituto

Tecnológico de Estudios Supe-

riores de Monterrey

pilar.arroyo@itesm.mx

María de Lourdes

Cárcamo Solís

Profesora titular, Universidad

Michoacán de San Nicolás de

Hidalgo

carcamo.lulu@gmail.com

\section{Resumen}

Este trabajo explora el efecto que la globalización ha tenido sobre las acciones tomadas por proveedores y compradores para mejorar las capacidades productivas y administrativas de los productores locales en las ramas industriales automotriz y textil y de la confección. La primera rama ha mantenido su situación de competitividad y aportación a la economía mexicana, mientras que la segunda ha perdido competitividad frente a los proveedores extranjeros. Debido a ello, fue importante analizar cómo cada rama ha abordado el problema de mejorar las capacidades de los proveedores. Después de analizar múltiples casos, se identificaron diferentes esquemas para el desarrollo de proveedores; una de las diferencias más destacadas fue la participación activa y directa de las firmas líderes de la cadena automotriz, lo que contribuirá a explicar el diferencial en competitividad entre las dos ramas.

Palabras clave: comercio internacional, desarrollo de proveedores, rama automotriz, rama textil y de la confección.

No. 228, mayo-agosto 2009: 105-126 


\title{
Comparative study about supplier development in two industry branches: automotive and textile \& apparel
}

\begin{abstract}
This work explores the effect that globalization has had over the actions deployed by suppliers and buyers to improve the capabilities of local producers in the automotive and textile \& apparel branches. The automotive branch has maintained its competitiveness and contribution to the Mexican economy, while the textile \& apparel branch has lost competitiveness against international producers; therefore it was relevant to analyze how each branches has faced the problem to improve the supplier's capabilities. The analysis of multiple case studies allowed the identification of different actions and structures for supplier development programs; one of the most outstanding differences between the programs in the two sectors is the active and direct participation of the leader firms in the automotive supply chain, this difference helps in explaining the competitiveness differential between both branches.
\end{abstract}

Keywords: international commerce, supplier development programs, automotive and apparel branches.

\section{Introducción}

La globalización ha contribuido al incremento de la compra internacional y a fomentar la competencia entre regiones geográficamente distantes para abastecer a las grandes empresas compradoras que dominan los mercados de mayor consumo. La tendencia en el abasto global ha dado como resultado fuertes presiones sobre los productores para que mejoren su competitividad, la cual depende en gran medida de las capacidades de los proveedores de la empresa (Modi y Mabert, 2007). Cuando las competencias de sus proveedores son deficientes, la empresa compradora tiene las siguientes opciones: a) identificar y seleccionar nuevos proveedores en otras regiones, b) evaluar y retroalimentar a los proveedores potenciales, dejándoles que tomen la iniciativa de mejorar sus capacidades y ofreciéndoles el incentivo de compras futuras, o c) implementar programas de capacitación y apoyo para que los proveedores mejoren su desempeño (Sánchez-Rodríguez et al., 2005).

Debido a lo anterior, el objetivo de este trabajo es explorar las actividades que empresas compradoras y fabricantes en las ramas industriales automotriz y textil y de la confección han implementado para mejorar las capacidades y el desempeño 
de los proveedores con el fin de enfrentar la tendencia globalizadora. En la primera sección se describe la contribución económica de estas dos ramas en la economía mexicana; en particular, se resalta cómo la competencia global y los acuerdos de comercio internacional han afectado la competitividad de los productores en ambas ramas. Mientras la primera hace un uso más intensivo de la tecnología y ha mantenido su situación de competitividad y aportación a la economía mexicana, la segunda al tener procesos que son más intensivos en el uso de mano de obra ha perdido competitividad frente a los proveedores extranjeros que ofrecen menores costos para sus productos. La situación de las dos ramas es contrastante, por lo que resulta importante analizar en qué forma los proveedores en cada una de ellas han abordado el problema de sostener su posición en el mercado global a través de la mejora de sus capacidades. En la siguiente sección se describe la metodología empleada para identificar los esquemas de desarrollo de proveedores vigentes en cada rama. En la tercera sección se categorizan los distintos mecanismos para mejorar las capacidades de las empresas en ambas ramas, con el fin de emplear esta clasificación posteriormente e identificar aquellos mecanismos que se utilizan en cada una para asegurar la competitividad de los productores locales. Finalmente, se presentan las conclusiones en la última sección.

\section{Metodología}

Debido a la falta de descripciones conceptuales sobre cómo las empresas participantes en cadenas de abasto globales enfrentan el reto de mejorar las capacidades de los proveedores regionales y cuáles son las diferencias operacionales entre ambas ramas distintas, este trabajo se basó en métodos cualitativos y datos secundarios para recopilar la información necesaria y de esta manera elaborar una representación de los mecanismos existentes para el desarrollo de proveedores, así como para identificar a las organizaciones que los promueven. En este trabajo los métodos cualitativos, dada su naturaleza exploratoria y sus propósitos de comprensión (Yin, 2003), resultaron convenientes para indagar sobre los métodos, prácticas y acciones desplegadas por las empresas, ya sea proveedores o clientes, para asegurar las competencias de la base de proveedores.

El primer paso del análisis de las condiciones en las que se proponen iniciativas para el desarrollo de proveedores fue realizar una revisión de la situación económica y de competitividad de las ramas automotriz y textil y de la confección para comprender las motivaciones de las empresas para perfeccionar sus capacidades. En una siguiente fase se recopiló información primaria a través de entrevistas per- 
sonales no-estructuradas con gerentes generales, gerentes de planta, gerentes de compra y administradores de centros de capacitación de apoyo a proveedores. Las entrevistas a fondo permitieron examinar las vivencias de varias empresas que han asumido una posición activa en la mejora de competencias según las demandas de su rama. Todos los entrevistados son profesionales con experiencia en la implementación de actividades encaminadas a mejorar las capacidades de su propia empresa o las de sus proveedores. En esta fase se realizaron un total de diez entrevistas (dos de ellas fueron entrevistas conjuntas en las que participaron dos ejecutivos de la misma empresa) que incluyeron a gerentes de primer nivel de las siguientes organizaciones:

Rama textil y de la confección. Entrevistas con la emprendedora que fundó Aztex S.A. de C.V., así como con el director general de esta empresa que opera como agente mercantil o trader. Entrevistas con el gerente general y el gerente de operaciones de la manufacturera Original Mexican Jeans Co.; y entrevista con el gerente general de una empresa fabricante de encajes cuyo nombre se omite por solicitud de la empresa.

Rama automotriz. El total de entrevistas realizadas con organizaciones que operan o apoyan iniciativas de desarrollo dentro de esta rama fueron seis. Tres de las entrevistas se realizaron en empresas que operan como proveedores tier-one de las armadoras automotrices y que serán denominadas como proveedor A, proveedor $\mathrm{B}$ y proveedor $\mathrm{C}$ por razones de confidencialidad. En el caso de los proveedores A y B, las entrevistas se realizaron con los gerentes de compras de cada empresa; para el caso del proveedor C, la entrevista se realizó con el gerente general de la planta ubicada en Durango. Los tres proveedores son compañías multinacionales; el proveedor A figura entre los proveedores más diversificados dentro de la rama automotriz e inició operaciones en México en 1991; en el 2007 este proveedor contaba con 241 plantas de manufactura y 62 centros de ingeniería y desarrollo ubicados principalmente en Europa y Norteamérica. El proveedor B cuenta con un total de 205 plantas; en América las plantas de esta empresa se ubican en Brasil, Estados Unidos y México; asimismo, las operaciones de esta empresa en nuestro país iniciaron en 1982. Este proveedor fabrica productos para tres mercados y uno de ellos es la rama automotriz, la cual contribuye con el $27.2 \%$ de las ventas totales que registra esta empresa en el nivel mundial. Por su parte, el proveedor $\mathrm{C}$ cuenta con plantas de manufactura ubicadas en 87 sitios estratégicos localizados en 38 países de seis continentes y ocupa el $14^{\circ}$ lugar de ventas (en dólares) entre los proveedores tier-one de la rama automotriz; en México el proveedor C abrió sus primeras instalaciones en Chihuahua en 1978. 
De igual manera, se realizaron dos entrevistas más, una con el director general Centro de Desarrollo para la Industria Automotriz Mexicana (CeDIAM) y otra con el asesor académico del Cluster Automotriz del Estado de México; estas dos organizaciones vinculan a los proveedores con las empresas líderes de la rama automotriz y les apoyan para que alcancen los niveles de desempeño requeridos. La sexta entrevista fue con el administrador del programa para el desarrollo de proveedores de una armadora automotriz que opera en México desde 1961 y que figura entre las cinco compañías automotrices líderes mundiales, reportando una participación en el mercado del 9.1\%.

Para recopilar la información se elaboró una guía de entrevista que fue adaptada según el tipo de empresa (proveedor, comprador o administrador de un programa de desarrollo); además, la entrevista fue guiada por una de las dos autoras del trabajo y asistida por la otra autora. Las entrevistas fueron grabadas, previa autorización del entrevistado, y ambas investigadoras tomaron notas individuales durante cada entrevista. Se obtuvo información adicional sobre el programa de desarrollo de proveedores de la armadora a través del análisis de documentos impresos proporcionados por el administrador del programa para desarrollo de proveedores durante su entrevista. También se obtuvo información suplementaria de las actividades de apoyo a proveedores que realizan el CeDIAM y el Cluster Automotriz del Estado de México a través de documentos y de la consulta de las páginas web: www.itesm.mx/rzc/cediam (CeDIAM) y www.clusterautomotriz.com; las páginas web de los tres proveedores tier-one también fueron revisadas. De igual forma, se obtuvo información secundaria a través de la participación de ambas autoras en los foros de discusión sobre la problemática que enfrentan las dos ramas y la propuesta de estrategias para su competitividad. Estos foros incluyen la "Iniciativa Ayatli para refuerzo de la competitividad de la cadena fibras-textil-vestido" (www.ayatli. com), promovida por la Secretaría de Desarrollo Económico (Sedeco) en diciembre de 2007 y la quinta y sexta ediciones del evento sobre intercambio de mejores prácticas y experiencias de la industria automotriz "AKJ Automotive Mexico" (www.tol.cem.akj) celebradas en octubre de 2007 y 2008.

Dada la importancia de la calidad de los datos para la investigación interpretativa, todas las entrevistas fueron transcritas; las transcripciones fueron analizadas por separado por ambas autoras y comparadas contra las notas tomadas durante su realización. Las repuestas proporcionadas por los entrevistados fueron validadas con la información expuesta abiertamente en los foros de discusión y con la que aparece en las páginas web de las empresas. La credibilidad de los datos fue asegurada 
al analizar la congruencia entre la interpretación sobre motivaciones y actividades para desarrollo de proveedores que realizaron por separado cada una de las autoras. Cabe aclarar que cuando hubo discrepancias en la interpretación, se recurrió a las transcripciones y se analizó el significado de las respuestas proporcionadas por los entrevistados, para lo cual se apoyó en la literatura.

\section{Importancia económica de las ramas industriales que se estudian}

\section{a) Rama textil y de la confección}

La rama textil y de la confección está constituida por varios subramas: fibras e hilos, que pueden ser de origen natural o químico (fibras artificiales y sintéticas); textiles (tejido, pie y trama, telas no tejidas, cintas, encajes, etc.); confección y acabado de prendas.

De acuerdo con los datos de la Secretaría de Economía ${ }^{1}$, a comienzos del nuevo milenio esta rama constituyó la cuarta actividad más dinámica de la industria manufacturera, generó el 1.2\% del PIB nacional y el 7.1 del PIB manufacturero. De 1994 a 2007, el valor de la exportación de esta rama se incrementó a un ritmo promedio anual del $3.7 \%$, contribuyendo con un 1.6\% al PIB de exportación. Para finales de 2000, México era el primer exportador de prendas de vestir a los Estados Unidos registrando 8.7 billones de dólares en 2000, con respecto a los 0.709 billones de dólares en 1990. Sin embargo, esta tendencia positiva se revirtió a partir de 2001 debido a la desaceleración de la economía estadounidense y a la competencia de los proveedores internacionales, mostrando un decremento promedio del $11 \%$ durante el periodo 2001 a 2007, según datos del INEGI. ${ }^{2}$

México ha firmado cerca de 15 acuerdos comerciales en el nivel internacional con más de 40 países, el más importante es el Tratado de Libre Comercio de América del Norte (TLCAN) que ha reducido el costo de los productos al eliminar aranceles, lo que hizo más atractivo al país para el abasto internacional en ramas como la textil y de la confección, automotriz y electrónico. Antes del TLCAN, los productores nacionales de la industria textil y de la confección estaban enfocados a atender la demanda doméstica o eran maquiladores, la ratificación del TLCAN en 1994 contribuyó a que firmas líderes como Calvin Klein, Liz Clairborne, Wal-Mart, JCPenny

${ }^{1}$ Ver http://www.economia.gob.mx

${ }^{2}$ Ver la página del Instituto Nacional de Estadística, Geografía e Informática (INEGI): http:www.inegi.gob.mx 
y Sears buscaran abastecerse en México, contribuyendo al crecimiento económico, la mejora de las capacidades productivas de los proveedores y el aumento de las exportaciones (Bair y Gereffi, 2002: 34). Las empresas de esta rama industrial se han concentrado en la zona central del país (Distrito Federal, Estado de México, Puebla y Guanajuato), en el norte (Comarca Lagunera y Chihuahua) y en los estados de Aguascalientes y Yucatán.

En el aspecto interno, las empresas de esta rama no sólo tienen capacidades limitadas para ofrecer servicios de "paquete completo", sino que además enfrentan problemas de poca flexibilidad para fabricar productos "tropicalizados", falta de información oportuna sobre preferencias del consumidor y tendencias en la moda internacional e insuficientes competencias para el diseño y la innovación. En cuanto al aspecto externo, las empresas mexicanas de esta rama industrial enfrentan los problemas de créditos limitados debido a su alto riesgo; deficiente desarrollo industrial que se aprecia en aspectos específicos como el bajo uso de la tecnología; bajo costo laboral de los fabricantes ubicados en los países asiáticos (China, India, Bangladesh y Pakistán, entre otros) y de la Cuenca del Caribe; contrabando de prendas de vestir de bajos precios; bajos aranceles a las prendas fabricadas en China -México aplica el 10\% de arancel y los EUA aplican un 25\% - y los nuevos acuerdos comerciales o en proceso que México tiene con China, Nueva Zelanda, Australia y Corea del Sur que se espera generen un mayor desequilibrio en la balanza comercial. Estos factores ejercen una mayor presión sobre las empresas de la rama textil y de la confección que requieren diseñar estrategias competitivas para conformar redes de producción mundiales capaces de producir grandes volúmenes a bajos costos, o reposicionarse como empresas flexibles y con desarrollo tecnológico capaces de producir pequeños lotes de productos de mayor valor agregado y en tiempos de entrega cortos (Knappe, 2005).

\section{b) Rama automotriz}

Está constituido por dos subramas: autopartes e industria terminal; la primera se dedica a la producción de partes y componentes y está integrada por grandes empresas fabricantes de equipo original (OEM, Original Equipment Manufacturer por sus siglas en inglés) como Magna, Bosch y Delphi, pero también por pequeñas y medianas empresas que operan en los niveles más bajos de la cadena productiva que lideran las oEM. La subrama de producto terminal se enfoca al ensamble de vehículos y está constituida por las grandes armadoras automotrices. 
Según el reporte de la Organización Internacional de Productores de Vehículos Automotores, México ocupó la décima posición como productor, con lo que ha avanzado sobre países como Inglaterra y España. Hay tres regiones geográficas donde se concentra la mayor actividad productiva automotriz: la parte central del país, ubicada en México, Puebla, Morelos y Tlaxcala; la zona del Bajío, en los estados de Aguascalientes, San Luis Potosí, Querétaro, Jalisco y Guanajuato; y la zona norte que comprende Baja California, Sonora, Coahuila, Nuevo León y Chihuahua.

De acuerdo con información consultada en la página del INEGI, ${ }^{3}$ la subrama de vehículos terminados tuvo un crecimiento promedio anual de $13.4 \%$ en el valor de las ventas, y un $8.3 \%$ en el número de unidades vendidas al exterior durante el periodo de 1994 al 2007. Este comportamiento muestra que la industria automotriz es una de las principales ramas a través de los cuales México se ha integrado al dinamismo del comercio internacional. Sin embargo, a partir de 2001 la recesión de la economía norteamericana contribuyó a la disminución de las exportaciones de vehículos, las cuales registraron una notable recuperación hasta 2005. El aumento del contenido ${ }^{4}$ nacional de $62.5 \%$ para los vehículos producidos en México, más la firma de nuevos acuerdos comerciales, ha provocado la atracción de armadoras que invitan a sus proveedores internacionales a ubicarse en México o promueven la mejora de las capacidades de los productores nacionales para integrar su base de proveedores.

Por su parte, la subrama de autopartes es la segunda exportadora después de la industria de manufactura de productos terminados con un crecimiento promedio anual de $9.6 \%$ en el valor de sus exportaciones durante el periodo 1994-2007. Sin embargo, esta subrama también registró en el comienzo del milenio una drástica caída de sus exportaciones, recuperándose hasta 2002. Si bien la firma del TLCAN consolidó la orientación exportadora de la subrama de automóviles, para la subrama de autopartes significó una contracción. La baja competitividad de los productores de autopartes (baja productividad, calidad deficiente y falta de modernización en sus sistemas de producción y en mano de obra calificada) se asocia con la balanza comercial negativa de la subrama y la desaparición de fabricantes nacionales de autopartes, sólo $20 \%$ de los fabricantes de autopartes concentran el $55 \%$ de la producción nacional y están en posibilidades de enfrentar la apertura comercial (Vicencio, 2007).

\footnotetext{
${ }^{3}$ Ver la página del Instituto Nacional de Estadística, Geografía e Informática (INEGI): http:www.inegi.gob.mx

${ }^{4}$ Piezas de producción local que debe contener un auto.
} 
En contraste con la rama textil y de la confección, para la cual la apertura comercial y la falta de apoyos gubernamentales han contribuido a su contracción económica, la rama automotriz — considerada estratégica para el desarrollo de México- ha contado con apoyos gubernamentales continuos (Vicencio, 2007) enfocados a regular y desarrollar la industria automotriz mexicana, como sucedió en 1962; promover su competitividad aumentando las exportaciones, como aconteció en 1972; lograr la modernización de la industria automotriz, como se planteó en 1989; mantener la inversión extranjera y autorizar la entrada de nuevas empresas fabricantes de vehículos, así como reducir los costos de las importaciones, que se dio en 2003.

\section{Mecanismos para el desarrollo de proveedores}

Las crecientes demandas de los consumidores, la competencia global y la necesidad de tiempos cortos para la introducción de nuevos productos demandan mayores capacidades técnicas, administrativas y de servicio para los proveedores de empresas líderes de cadenas de abastecimiento. Krause et al. (2000: 34) definen el desarrollo de proveedores como "cualquier actividad promovida por la firma compradora para mejorar ya sea el desempeño o las capacidades del proveedor con el fin de satisfacer las necesidades de abasto de corto y largo plazo de la empresa compradora." Múltiples actividades pueden incluirse en un programa de desarrollo de proveedores, que son clasificadas por el mismo Krause et al. (2000: 36-38) como actividades externas ${ }^{5}$ e internas. Las actividades externas incluyen la evaluación de proveedores, el impulso a la competencia y el empleo de incentivos en la forma de premios y promesas de una mayor compra; entre estas estrategias figuran la competencia entre proveedores, la evaluación de proveedores y el uso de incentivos en la forma de compras regulares o de mayores volúmenes. Por su parte, las actividades internas implican un mayor involucramiento de la empresa compradora e incluyen capacitación, visitas in situ, asesoría directa para la identificación y solución de problemas y formación de equipos que colaboran para el diseño de nuevos productos, así como la propuesta conjunta de proyectos de mejora. Si bien para autores como Krause et al. (2000) un programa de desarrollo de proveedores involucra la participación de la empresa cliente, Helper y Kiehl (2004: 96-97) identifican los siguientes dos tipos de mecanismos disponibles para la mejora de capacidades de los proveedores:

\footnotetext{
${ }^{5}$ Krause et al. emplean los términos externalized e internalized, los cuales fueron traducidos al español como actividades externas e internas porque el cliente se sirve ya sea del mercado externo o de sus recursos internos para estimular la mejora del proveedor.
} 
1) Mecanismos disponibles en el mercado. Estos métodos se refieren a la contratación de servicios especializados por parte del proveedor, se incluyen servicios de consultoría, la adquisición de materiales impresos o grabados, la contratación de expertos y cursos de capacitación especializados.

2) Mecanismos no-disponibles en el mercado. Estos métodos incluyen todas las actividades de desarrollo destinadas a proveedores seleccionados que promueven directamente las empresas líderes de la cadena, las asociaciones industriales, los consorcios de proveedores o las agencias de gobierno.

Los mecanismos de apoyo a proveedores en cada categoría tienen ventajas y desventajas. En el caso de los disponibles en el mercado, sus limitaciones principales son su costo, el cual absorbe el proveedor y su enfoque de corto plazo. Sus ventajas principales son que dependen poco del cliente y promueven la adquisición de capacidades genéricas que el proveedor puede transferir cuando surte a múltiples clientes. Los métodos no-disponibles en el mercado tienen como ventajas: contar frecuentemente con subsidios, el conocimiento que se transfiere está dirigido a la aplicación inmediata y enfocado a lograr la certificación del proveedor ante empresas líderes. El contenido de los programas involucra conocimientos especializados que se consideran propietarios de la empresa líder y, por tanto, no están disponibles a través de otras fuentes. Las desventajas principales son que los mecanismos tienden a ser "cerrados" — esto es, son accesibles sólo a las empresas vinculadas con la organización promotora- y su contenido se enfoca en atender las necesidades específicas de un cliente.

\section{Programas de desarrollo de proveedores en las ramas que se estudian}

\section{a) Rama textil y de la confección}

Bair y Gereffi (2002: 34-36) distinguen como empresas líderes en cadenas productivas de bienes de consumo o cadenas de abastecimiento impulsadas por el consumidor a las grandes empresas de venta al menudeo, a los dueños de marca y a los agentes comercializadores (incluyéndose en este rubro a los intermediarios que facilitan la exportación). Estas empresas figuran como empresas focales porque dominan las actividades de mayor valor en esta cadena - diseño, mercadotecnia y distribución — manteniendo su posición de liderazgo a través del control del capital y la información sobre el mercado (Gereffi, 2001: 1620-1622). El proceso de producción de esta industria es intensivo en mano de obra, lo que da lugar a barreras de entrada relativamente bajas para los productores, por lo que predomina la 
libre competencia y las empresas líderes pueden elegir entre múltiples productores y cambiar fácilmente de zona de abasto.

La información proporcionada por las empresas entrevistadas dentro de la rama textil y de la confección en cuanto a información y acciones de mejora promovidas por sus compradores concuerda con el marco teórico propuesto por Gereffi (2001: 1618-1622) en el sentido de que las empresas líderes en la cadena limitan sus actividades de desarrollo de proveedores a la supervisión del producto terminado y a la observación del cumplimiento de políticas que aseguren la responsabilidad social del comprador. Cabe aclarar que las empresas entrevistadas a fondo, como las que participaron en las reuniones de la iniciativa Ayatli, reconocieron que para mantenerse en el mercado han tenido que asumir una actitud más proactiva y tomar directamente la responsabilidad de mejorar sus capacidades para la investigación de mercados, la innovación en producto y el desarrollo de relaciones de asociación con fabricantes críticos (textileros y acabadores).

Los productores de ropa y accesorios utilizan principalmente mecanismos disponibles en el mercado para mejorar sus capacidades, que incluyen la "compra" de capacitación a terceras partes como universidades (e.g. Tecnológico de Monterrey-ITESM o la Universidad Iberoamericana), cámaras industriales y de comercio (e.g. Cámara Nacional de la Industria del Vestido), dependencias gubernamentales (e.g. Secretaría de Desarrollo Económico y Bancomext) y la contratación de servicios de empresas consultoras. Otro mecanismo de adquisición de conocimientos es la contratación de recurso humano experto, ya sea ex empleados con experiencia laboral en empresas líderes de la rama o profesionales en áreas como mercadotecnia y logística en las que requieren introducir nuevas competencias. A continuación se sintetizan las acciones específicas de las tres empresas entrevistadas, las cuales corroboran estas observaciones generales. Asimismo, cabe indicar que estas empresas tienen una posición distintiva en la cadena fibras-textil-vestido: un proveedor especializado en la producción de insumos, una empresa con capacidad de producción de paquete completo y un agente intermediario que subcontrata la manufactura para ofertar producción de paquete completo.

La primera de las empresas entrevistadas fabrica encajes, ya sea para productos de decoración o prendas de vestir, y opera como proveedor de empresas que ofrecen producción de paquete completo como Íntima (ubicada en Atlacomulco) y empresas dueñas de marca (Avon y Fuller). Este proveedor ha reenfocado su estrategia, pues de estar centrada en los precios ha pasado a orientar la creación de valor para 
el cliente; esta estrategia se basó en investigación de mercados, innovación en producto y reducción de ciclos de producción y entrega. Para desarrollar estas capacidades, el proveedor ha utilizado los siguientes mecanismos: a) gestión de financiamiento ante agencias gubernamentales (Promex y la Dirección de Exportaciones de la Secretaría de Economía) para realizar estudios de investigación de mercados y atender a cursos de capacitación; b) apoyo de instituciones educativas, que por un costo simbólico realizan los estudios de investigación de mercados necesarios y c) contratación de personal con conocimientos sobre mercadotecnia, transferencia de tecnologías para el desarrollo de nuevos productos y administración de inventarios. A través de sus esfuerzos internos, la empresa introduce hasta 15 nuevos productos por mes (anteriormente eran tres), sus muestrarios atienden a las tendencias en moda europea y sudamericana, además de hacer mejor promoción de sus productos. De parte de sus clientes, la empresa sólo recibe retro-alimentación e incentivos equivalentes a un mayor volumen de compra.

El intercambio de conocimientos y experiencias entre las empresas que integran las cadenas productivas de la rama textil y de la confección no se identificó como un mecanismo vigente para la mejora de capacidades. Sin embargo, la formación de alianzas estratégicas entre fabricantes locales e internacionales sí resultó ser un mecanismo relevante, como ilustra el caso de la empresa Original Mexican Jeans Company (OMJC) $)^{6}$ ubicada en la Comarca Lagunera y líder en la producción de pantalones de mezclilla, que ha desarrollado capacidades logísticas, de diseño y de desarrollo de productos. OMJC inició como confeccionista de prendas; los textiles, avíos y piezas cortadas eran proporcionados por su socio internacional que se encargaba de los procesos de lavado y acabado. Eventualmente, y debido a la reducción de los costos de producción en México, el proceso de producción completo se transfirió a OMJC, que desarrolló nuevas capacidades de manufactura y amplió sus instalaciones para incluir un centro de corte, cuatro plantas de costura y un centro de lavado.

Para responder a las demandas de sus clientes internacionales (JC Penny, Gap, Old Navy, Goals, Academy, Harley Davison, entre otras) OMJC mejoró sus actividades logísticas abriendo un centro de almacenaje en 2000 e implementando un programa de administración del inventario del cliente (VMI, Vendor Management Inventory)

${ }^{6}$ OMJC fue fundada en 1992 como coinversión de la familia mexicana Martín-Soberón y la empresa norteamericana Aalfs Manufacturing. El interés de Aalfs fue producir en México manteniendo el control a través de la formación de una sociedad con un productor local con capital y experiencia. Inicialmente, Aalfs participó con el 40\% de las acciones, pero en marzo de 2007 esta empresa hizo la oferta de adquisición de OMJC, actualmente está en proceso de transferirse a la compañía norteamericana. 
en 2003. Para 2004, creó un centro para la investigación sobre acabados y desarrollo de diseños. Estas capacidades adicionales de producción, logística y diseño fueron desarrolladas por OMJC con el apoyo de Aalfs Manufacturing y sin la intervención directa de sus clientes, quienes circunscribieron su apoyo a la supervisión regular de las prácticas de producción de su proveedor. Por su parte, omsc como empresa líder en la rama, no promueve programas de desarrollo ni intercambio de conocimientos con otros productores, excepto por empresas consideradas socios estratégicos como Parras-Cone, empresa textil líder, y dos empresas ubicadas en Torreón, Coahuila, a quienes OMJC subcontrata los servicios de lavado de prendas para cubrir su falta de capacidad.

Con el fin de lograr la flexibilidad y la velocidad requerida para atender la demanda volátil de su mercado, las empresas líderes de las cadenas textil y del vestido requieren administrar una compleja red de proveedores (Masson et al., 2007). Cuando estas empresas líderes no tienen las capacidades o el interés para realizar la coordinación de la red productiva, utilizan los servicios de agentes intermediarios o traders, quienes son populares en Asia (Bair y Gereffi, 2002: 37), aunque apenas están surgiendo en otras regiones. Estos agentes intermediarios capitalizan sus conocimientos sobre las capacidades productivas, políticas comerciales y cultura de negocios local para ofrecer un paquete de servicios variado y complejo, que va desde facilitar los trámites de exportación de prendas terminadas (servicio de broker) hasta servicios de producción de "paquete completo" (Bitran y Arroyo, 2007: 31-52). El tercer caso de análisis corresponde a Aztex Trading, S.A de C.V un intermediario mexicano que sirve a clientes nacionales (e.g. Palacio de Hierro y Julio) e internacionales (e.g. Hugo Boss y Grupo Indetex).

Aztex coordina casi todas las actividades de valor en la cadena textil y del vestido (excepto la comercialización) desde la preparación de catálogos y muestras, hasta la entrega de pedidos de composición compleja en las instalaciones del cliente. Este agente intermediario cuenta con oficinas en 16 zonas productoras, desde donde supervisa y orienta a los productores a quien subcontrata. Aztex apoya la promoción de diseños textiles a través de la administración de una teleteca (directorio de fabricantes de fibras y textiles) que cuenta con el soporte de la Secretaría de Economía. Al realizar esta función, el agente intermediario acumula y renueva sus conocimientos sobre textiles y fibras nacionales, que capitaliza cuando genera catálogos y surte órdenes para sus clientes. Aztex obtiene también información continua sobre el mercado de la moda, que transfiere a los textileros a quienes ofrece además retroalimentación sobre sus nuevos productos y recomienda las tec- 
nologías requeridas para desarrollar acabados de moda. Esta información sobre el mercado la transfiere también a los confeccionistas de su red de abasto, apoyándoles en la adaptación de los diseños a las preferencias del mercado nacional, para lo cual supervisa la calidad de los productos y asume la responsabilidad de su movimiento y entrega. A través de la transferencia continua de información, la supervisión y la retroalimentación, Aztex asume una posición más activa en el desarrollo de los productores de la cadena fibras-textil-vestido que la que tienen otras empresas líderes de la cadena.

El diagrama de la figura 1 resume gráficamente el análisis de los casos, también se describen los mecanismos utilizados por los productores en las cadenas fibrastextil-vestido para mejorar sus capacidades. Las líneas continuas representan flujos de información y conocimientos que son adquiridos en el mercado por iniciativa de los proveedores, principalmente vía la intervención de terceras partes. En el diagrama también se muestra la contribución al desarrollo de capacidades que se da a través de alianzas estratégicas.

Figura 1

Cadenas impulsadas por el consumidor: Mecanismos para la mejora de capacidades a partir de la transferencia de conocimientos e información

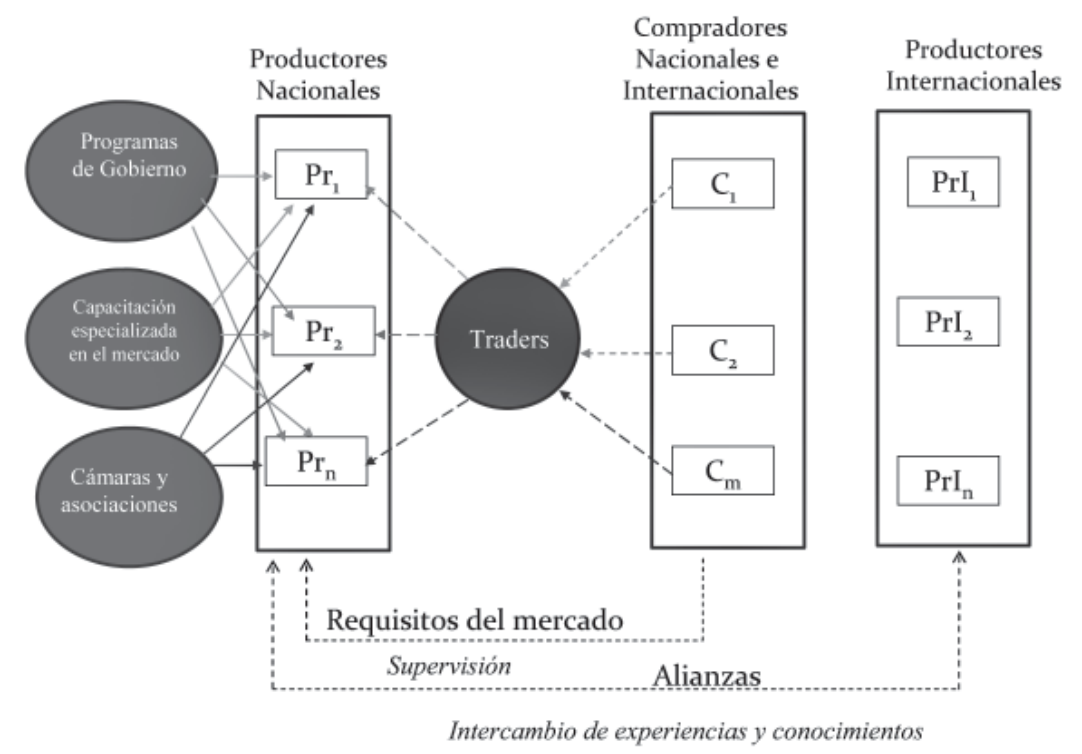

Fuente: elaboración propia con base en la información recopilada en este estudio 


\section{b) Rama automotriz}

Esta rama es representativa de lo que Gereffi (2001: 1618) define como cadena global (de abastecimiento) impulsada por el producto. La rentabilidad en este tipo de cadena proviene de la innovación en el productor y la tecnología, las mejoras en los procesos productivos y la coordinación interfirma. La estructura de la cadena es jerárquica, con flujos verticales de conocimiento, altas barreras de entrada para nuevos proveedores, además, los líderes de la cadena son empresas de manufactura que protegen sus capacidades técnicas y productivas a través del control de la información sobre tecnologías de producto y proceso; la participación en el diseño del producto está limitada a sus socios estratégicos (Bagchi y Skjoett-Larsen, 2003: 5). Los programas de desarrollo de proveedores, altamente estructurados y liderados por las empresas focales, resultaron ser el mecanismo predominante según el análisis de la información proporcionada por las empresas entrevistadas dentro de esta rama, lo que concuerda con la literatura sobre programas globales de desarrollo de proveedores como Toyota Production System, BP de Honda y V Production Way de Nissan (Sako, 2004).

El administrador del programa de desarrollo de proveedores de la armadora automotriz declaró en su entrevista que el programa está orientado hacia la recertificación del proveedor a través de las siguientes acciones: 1) selección de participantes en términos de su importancia económica (volumen de ventas) para la armadora y su capacitación genérica en el modelo productivo propietario de la automotriz, 2) capacitación técnica en manufactura y logística, orientada hacia mejoras en proceso, aseguramiento en calidad de manufactura y cumplimiento en la entrega, y 3) seguimiento de las actividades del proveedor en cuanto a las mejoras realizadas más auditorías periódicas por parte de la armadora. El programa contempla capacitación del personal del proveedor a varios niveles, incluyendo la alta gerencia. Los contenidos de la capacitación y la asesoría técnica in situ se orientan a que el proveedor asimile los conocimientos de manufactura propietarios de la automotriz, de tal manera que el proveedor contribuya a mejorar las capacidades de producción de la automotriz.

Para implementar el programa, la armadora subcontrató a una organización educativa (Instituto Tecnológico y de Estudios Superiores de Monterrey), que realiza la instrucción desde un formato teórico-práctico a cargo de empleados expertos y de asesores técnicos vinculados con la institución, quienes son capacitados y autorizados por la armadora. El esquema de capacitación incluye la formación de grupos 
interdisciplinarios e interfuncionales (calidad, ingeniería, diseño, control de la producción, ventas), capacitación tradicional, transferencia de las prácticas, técnicas de manufactura y calidad de la automotriz hacia el proveedor y capacitación en logística a empleados del proveedor que se consideran clave. La participación de la institución educativa como agente externo responsable de la administración del programa es controlada por la armadora y los conocimientos propietarios (cores) sólo son transferidos por el personal de la empresa a proveedores que están calificados como críticos en función del volumen de productos que surten a la armadora o a su potencial para surtir nuevos productos.

La participación de terceras partes público-privadas facilita la administración de los programas de desarrollo de proveedores y permite conjuntar esfuerzos. Evidencia de esto, se encuentra en el programa de desarrollo del cluster automotriz del Estado de México, en el cual se conjuntan los recursos humanos y capacidades de instituciones de servicios educativos con los recursos económicos que destina la Dirección General de Industria de la Secretaría de Economía, además de los conocimientos expertos ${ }^{7}$ de las empresas manufactureras líderes de la rama automotriz (OEM), tanto armadoras como proveedores tier-one. El programa anterior tiene como objetivos: a) vincular a las empresas líderes de la rama con productores que tengan el potencial para figurar como proveedores de la cadena automotriz, y b) gestionar apoyos para que estas empresas desarrollen las capacidades productivas, tecnológicas y logísticas requeridas por las oEM. Si bien la integración de proveedores de niveles inferiores (dos a cuatro) a la cadena automotriz resulta difícil debido a las usuales relaciones transaccionales entre las oEM y sus proveedores, la literatura cita casos de organizaciones público-privadas que diseñan y administran programas de desarrollo de proveedores. Uno de estos casos es el Consorcio de Capacitación y Extensión de Relaciones con Proveedores de Manufactura de Wisconsin (Wisconsin Manufacturing Extensive Partnership, WMEP), en el que participan seis importantes oEM que delinean las capacidades que los productores requieren desarrollar, el sistema de Colegios Técnicos de Wisconsin (Wisconsin Technical College) e instituciones gubernamentales regionales, estatales y federales que financian la asesoría técnica que da el WMEP (Whitford y Zeitlin, 2004).

Por otra parte, el CeDIAM es una organización soportada por una red nacional de cámaras de gobierno, empresas líderes de la rama automotriz que forman parte

\footnotetext{
${ }^{7}$ Cabe señalar que en administración se reconoce que hay aprendizaje organizacional, el cual es resultado de la
} implementación de rutinas y procesos de la empresa para utilizar los conocimientos de las personas. 
de su consejo ejecutivo e instituciones educativas (Instituto Tecnológico y de Estudios Superiores de Monterrey, Instituto Tecnológico y Estudios Superiores de Occidente, Instituto Tecnológico de Sonora y Universidad de Sonora). El centro de desarrollo industrial automotriz apoya en dos formas el desarrollo de proveedores regionales para que sustituyan a los extranjeros, principalmente estadounidenses y japoneses: 1) asiste al proveedor para que logre la certificación de las oEM mediante apoyos para el desarrollo de innovaciones tecnológicas con financiamiento del gobierno y 2) promueve la creación de nuevas empresas de base tecnológica. Dado que las empresas de la rama automotriz tienden a mantener relaciones de asociación sólo con sus proveedores estratégicos, el CeDIAM promueve un modelo en "cascada" que fomenta la colaboración de una empresa con sus proveedores directos (proveedor tier-one con proveedor tier-two, tier-two con tier-three, entre otros).

Las entrevistas con los gerentes de oEM que figuran como proveedores tier-one (A, B y C) reveló que sus actividades de desarrollo de proveedores son principalmente de tipo externo (Krause, 2000: 35) e incluyen estimular la competencia a través de la selección bajo múltiples criterios, y la evaluación continua a través de auditorías y certificaciones. Las empresas A y B ofrecen además cursos de capacitación, asesoría para la certificación y mentoría in situ para atender problemas particulares. En contraste, el proveedor $\mathrm{C}$, cuando surte a armadoras enfocadas a la calidad y con una cultura de colaboración, establece asociaciones con sus proveedores directos para implementar proyectos de mejora continua y desarrollar nuevos productos. Los programas de desarrollo de estos tres proveedores tier-one están vinculados con las iniciativas locales (modelo Sonora, cluster automotriz del Estado de México y desarrollo económico en Durango) que promueven la integración de la cadena automotriz y la mejora de capacidades de los productores regionales, lo que conjunta los esfuerzos de los gobiernos locales con los de estas OEM. Sin embargo, los programas de A y B se enfocan en el desarrollo de capacidades de nivel bajo a medio; es decir, se impulsa a los proveedores para que mejoren sus procesos, su productividad, su eficiencia y con ello logren la reducción de costos que resulte en una buena rentabilidad para la empresa líder. El proveedor C apoya este enfoque sólo cuando funge como proveedor de armadoras que demandan bajos costos para las que selecciona proveedores en niveles secundarios mediante licitaciones, un mecanismo basado en la competencia. Se concluye de este análisis que los intercambios de información y conocimientos que promueven estas tres empresas líderes de la rama automotriz son intermitentes, están circunscritos al contexto de proyectos específicos e involucran la transferencia sobre la filosofía de 
la empresa y la información sobre sus procesos de manufactura sólo cuando estos conocimientos permiten mejoras relevantes en productividad y calidad.

El desarrollo de proveedores a través de la adquisición de conocimientos de fuentes externas tanto con fines de lucro (empresas consultoras), como sin ellos (agencias gubernamentales, asociaciones de proveedores y cámaras industriales), se identificó también como mecanismo viable para el desarrollo de capacidades. Los consultores externos que contratan las empresas de la rama automotriz son ex empleados de las empresas líderes con una extensa base de conocimientos prácticos. La naturaleza del conocimiento que ofertan estos consultores no es crítica porque no involucra aspectos medulares sobre tecnología de producto o proceso, sino asesoría en actividades consideradas de soporte, como compras, identificación y negociación con proveedores y logística. En el caso de agencias gubernamentales o asociaciones industriales, la oferta de capacitación y desarrollo tiende a ser genérica y de un alto contenido de conocimiento explícito, por lo que el impacto de estos mecanismos disponibles en el mercado es menor que el que se alcanza a través de los programas controlados o apoyados por las empresas líderes.

El análisis de la información de todos los casos para esta rama apoya la proposición de que el desarrollo de proveedores en las cadenas automotrices implica un gran involucramiento de las empresas líderes, puesto que se transfieren experiencias y conocimientos calificados como propietarios o críticos para los productos y procesos automotrices; este tipo de conocimientos críticos son los que tienen el potencial de contribuir al desempeño de los proveedores. La participación de otras instituciones públicas y privadas crea una sinergia que — como en el caso más avanzado del consorcio de capacitación y extensión de relaciones de Wisconsinayuda a la buena ejecución del programa. El diagrama en la figura 2 describe estos hallazgos. La línea continua y con flecha en una sola dirección corresponde a flujos verticales de conocimientos, en tanto las líneas discontinuas con doble flecha representan intercambios de información entre organizaciones externas y оEм para estructurar los programas de desarrollo de proveedores regionales. El diagrama también considera los mecanismos de desarrollo disponibles en el mercado. 
Figura 2

Cadenas impulsadas por el productor: Mecanismos para la mejora de capacidades a partir de la transferencia de conocimientos e información

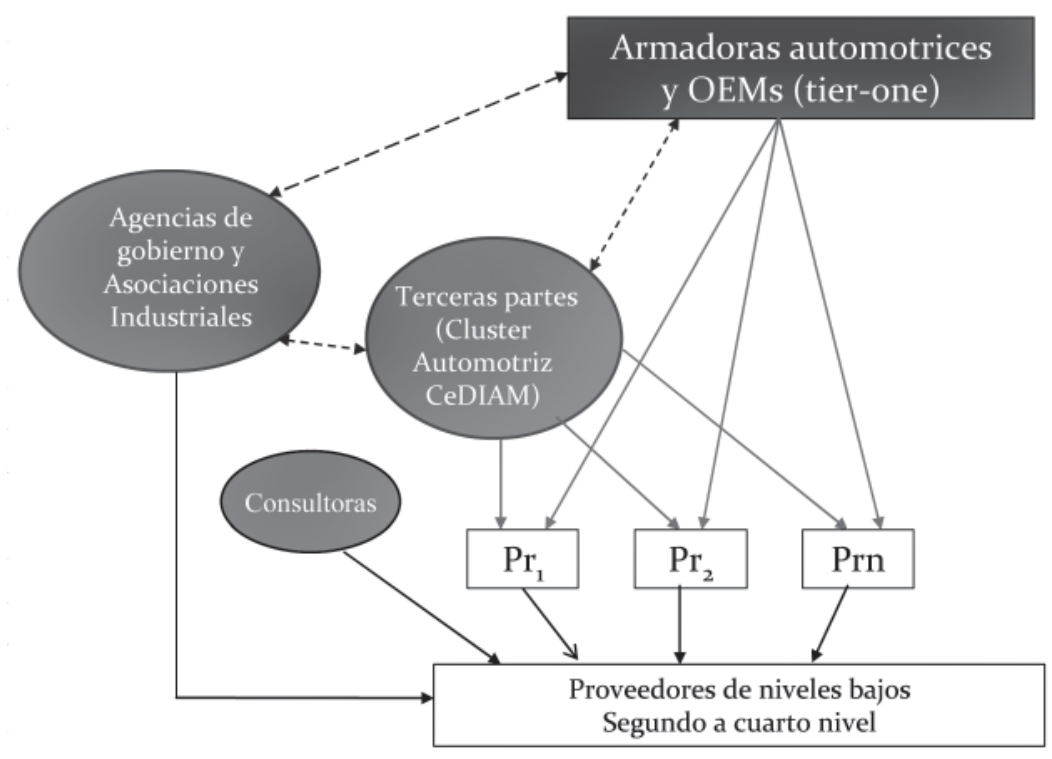

\section{CeDIAM= Centro para el Desarrollo}

Fuente: elaboración propia en base a la información recopilada en este estudio

\section{Conclusiones}

La rama textil y de la confección en México está caracterizada por una relativa entrada y salida de empresas, la producción es fragmentada y volátil, la rentabilidad se deriva en gran medida de los tratados comerciales, las cuotas limitantes y el valor de las marcas. La liberación del mercado y la sobreoferta productiva afecta a la rama textil y de la confección e impone a los productores la necesidad de incrementar su competitividad. Para ello, las empresas de esta industria han tenido que desarrollar nuevas capacidades productivas en función de la innovación en producto, la orientación al cliente y el establecimiento de relaciones de asociación con textileros y acabadores con el fin de integrar la cadena productiva. Las competencias que requieren desarrollar los productores de la rama deben permitirles evolucionar de una manufactura enfocada a la confección (maquila) de grandes 
volúmenes de prendas hacia un esquema de producción flexible de prendas no estandarizadas. Sin embargo, el análisis de los casos revela que los fabricantes de esta rama reciben apoyo limitado para enfrentar los cambios de su mercado teniendo que buscar sus propias alternativas de desarrollo. Las empresas líderes (dueños de marca, distribuidores y comercializadores) se limitan a las actividades de supervisión y evaluación; además, no tienen programas de mayor involucramiento que contribuyan al desarrollo de capacidades de los productores. Por otra parte, los programas gubernamentales de asistencia (e.g. la iniciativa Ayatli) son escasos, ofrecen capacitación genérica y dejan a los productores la responsabilidad de desarrollar los mecanismos de integración de su cadena y los canales de venta para el mercado local.

En contraste, en la rama automotriz, las empresas líderes - tanto armadoras como proveedores tier-one - se involucran directamente en el desarrollo de sus proveedores. En esta rama, el TLCAN en particular ha motivado que las oEM busquen desarrollar proveedores nacionales o invitar a sus proveedores internacionales a ubicarse en México, en lugar de recurrir al abasto global que favorecen las empresas líderes de la rama textil y de la confección. La inversión y participación de las OEM automotrices asegura que los programas brinden el know-how y la asistencia necesaria para que el proveedor pueda certificarse e integrarse a la cadena automotriz e incluso participar en otras actividades generadoras de valor aparte de la producción, como es el desarrollo de nuevos productos. Adicional a esta asistencia que brindan las empresas líderes, las iniciativas gubernamentales para apoyar a esta rama han sido más consistentes y orientadas a asegurar su competitividad; en el Estado de México - iniciativas como el cluster automotriz y la formación de organismos público-privadas como el centro de desarrollo para la industria automotriz- facilitan la vinculación de los proveedores que operan en niveles bajos (tier two a tier-four) con las oEM y conjuntan los esfuerzos del gobierno y la iniciativa privada para integrar a los productores regionales a la cadena automotriz.

Finalmente, podemos afirmar que ambas ramas industriales enfrentan los retos de un ambiente de negocios global, en el cual los acuerdos de comercio internacional y las políticas gubernamentales han tenido un efecto diferente sobre la integración de sus cadenas de abastecimiento. Aparte de estos elementos en el nivel macro, en el nivel de la cadena, este estudio identifica diferencias importantes entre los mecanismos para el desarrollo de proveedores (bajo vs. alto involucramiento y existencia de organizaciones de apoyo público-privadas), lo que contribuye a explicar las diferencias en competitividad entre las dos ramas. 


\section{Referencias}

ARGOTE, L. (2005). "Reflections on Two Views of Managing Learning and Knowledge in Organizations." Journal of Management Inquiry, Vol. 14 (1), pp. 43-48.

Bagchi, P. K. y T. SkJoett-Larsen (2003). ”Organizational Integration in Supply Chains: A Contingeny Approach." Global Journal of Flexible Systems Management, Vol. 3 (1), pp. 1-10.

BAIR, J. y G. GerefFi (2002). "NAFTA and the Apparel Commodity Chain.” En Free Trade and Uneven Development, Gereffi, Gary, Spener, David and Bair, Jennifer (editors). Philadelphia, PA: Temple University Press, pp. 23-48.

Bitran, G. R. y P. E. Arroyo (2007). "Coordination of Supply Chain Networks and the Emergence of Mini-Maestros" (12/2007). MIT Sloan Research Paper No. 4674-08, pp. 1-63. Disponible en sSRN: http://ssrn.com/abstract $=1082440$

GerefFI, G. (2001). "Shifting Governance Structures in Global Commodity Chains, with Special Reference to the Internet." American Behavioral Scientist, 44 (10), pp. 1616-1637.

HelPeR, S. y J. KieHL (2004). "Developing Supplier Capabilities: Market and Non-market Approaches. Industry and Innovation, Vol. 11 (1/2), pp. 89108.

KNAPPE, M. (2004). "Position paper: ITCs technical assistance approach for the Textile and Clothing Sector." En Clothing Europe: Comparative Perspectives on Trade Liberalization and Production Networks in the New European Clothing Industry, octubre. Disponible en línea http://www.unc.edu/ depts/europe/conferences/04/global/papers.htm).

Krause, D. R., T. V. Scannell y R. J. Calantone (2000). “A Structural Analysis of the Effectiveness of Buying Firms Strategies to Improve Supplier Performance." Decision Sciences, Vol. 31 (1), pp. 33-55. 
Masson, R., L. Iosif, G. MacKerron y J. Fernie (2007). "Managing Complexity in Agile Global Fashion Industry Supply Chains." The International Journal of Logistics Management, Vol. 18 (2), pp. 238-254.

Modi, S. B. y V. A. Mabert (2006). "Supplier Development: Improving Supplier Performance through Knowledge Transfer." Journal of Operations Management, Vol. 25, pp. 42-64.

SAKo, M. (2004). "Supplier Development at Honda, Nissan and Toyota: Comparative Case Studies of Organizational Capability Enhancement." Industrial and Corporate Change, Vol. 13 (2), pp. 281-297.

SÁnchez-Rodríguez, C., D. Hemsworth y Á. R. Martínez-Lorente (2005). "The Effect of Supplier Development Initiatives on Purchasing Performance: A Structural Model." Supply Chain Management, Vol. 10 (3/4), pp. 289-301.

Vicencio Miranda, A. (2007). "La industria automotriz en México: Antecedentes, situación actual y perspectivas." Contaduría y Administración, No. 221, enero-abril Facultad de Contaduría y Administración, UNAM, pp. 211-248.

Whitford, J. y J. Zeitlin (2004). "Governing Decentralized Production: Institutions, Public Policy, and the Prospects for Inter-firm Collaboration in US Manufacturing." Industry and Innovation, Vol. 11 (1/2), pp. 11-45.

YIN, R. K. (2003). Case Study Research. Thousand Oaks, CA: Sage Publications. 